\title{
Philip F. Stahel. Blood, Sweat and Tears: Becoming a Better Surgeon
}

\author{
Tfm Publishing Ltd; 1st Edition (15 February 2016), ISBN-10: 1910079278, ISBN-13: \\ 978-1910079270
}

\author{
I. Marzi $^{1}$
}

Published online: 27 June 2016

(C) Springer-Verlag Berlin Heidelberg 2016

"It takes 10 years to learn, 10 years to practice and 10 years to know when not to cut".

Philip Stahel, an experienced trauma and orthopedic surgeon, wrote a unique book every surgeon needs to read and think about! The idea behind this book is to open the eyes to become an even better surgeon: in technical aspects, in behavioral aspects and in respect to safety of our patients. In Blood, Sweat and Tears, Philip Stahel tells the reader very nicely and friendly and in a type of a narrative conversation the story of his personal surgical life, beginning with the status of a resident and resulting in a self-critical chairman of major University Orthopedic Department. His major aim is to open the eyes of any surgeon to reflect on their daily work to provide best care to their patients and to improve patient safety. The latter is of major interest to the author, who is in addition a strong advocate of patient safety, e.g., as Editor of the journal, Patient Safety in Surgery.

Step by step, Philip Stahel describes his personal experience of surgical responsibility: first, blood and sweat as surgical resident, followed by tears during decades of hard surgical experience. As a consequence, he nicely let the reader know that empathy is one of the key skills for a good surgeon, however, always under the assumption that sophisticated technical skills are crucial. Second, the author offers many options to be a better surgeon by discussing actual trends and providing strong examples of possible areas of improvement. Sound arguments for true awareness of complications, minimizing risks or solid indications are

I. Marzi

marzi@trauma.uni-frankfurt.de

1 Department of Trauma, Hand, and Reconstructive Surgery, Goethe-University Hospital, Theodor-Stern-Kai 7, 60590 Frankfurt/Main, Germany just some examples. Third, many aspects of being treated safely as a patient is discussed in detail; and current main stream procedures are questioned. One convincing example is an exemplary story that indicates that safety checklists are only working if they are adapted and lived by the team, and not artificially imposed. Fourth, the "pseudo-safety" of our current health care systems is disclosed: increasing formalism instead of personal responsibility are the real major threats of our system. At last, the solution to become a better surgeon comes out of the presented realistic and philosophical considerations; a credible self-control and leadership is essential, and the best way for optimal patient safety and avoidance of personal burnout.

If at all there can be anything criticized, there may a missing "chapter zero" for all the readers not being surgeons. In such a chapter, the real life of a surgeon, the tremendous work load, the real burden, and the personal responsibility might be brought to the attention of non-surgeon readers. This very interactive written book is dedicated to surgeons of all fields and will open the mind of every reader, since real surgeons understand everything written by this very honest author. Even if not every consequence and solution suggested by the author will represent a personal solution for every individual, each surgeon will adapt part of the daily standard work to be a better person after reading this book. Thus, this impressive book by Philip Stahel will lead us to being safer surgeons, and thereby, allow us to contribute to increasing patient safety. Therefore, as a department chair, I bought this book for all my staff members! 